\title{
Heart Rate Recovery at 1 Min after Exercise Is a Marker of Disease Severity and Prognosis in Chronic Thromboembolic Pulmonary Hypertension
}

\author{
Qi Jinn ${ }^{\mathrm{a}, \mathrm{b}}$ Xin $\mathrm{Li}^{\mathrm{a}}$ Yi Zhang ${ }^{\mathrm{a}}$ Zhihui Zhao ${ }^{\mathrm{a}}$ Qing Zhao $^{\mathrm{a}}$ Lu Yan $^{\mathrm{a}}$ Anqi Duan ${ }^{\mathrm{a}}$ \\ Qin Luo ${ }^{\text {a }}$ Zhihong Liu ${ }^{\mathrm{a}}$
}

${ }^{a}$ Center for Pulmonary Vascular Diseases, National Center for Cardiovascular Diseases, Fuwai Hospital, Chinese

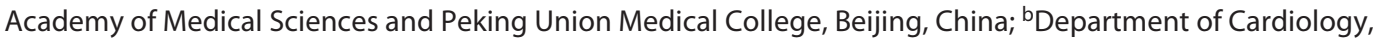
Shanghai Institute of Cardiovascular Disease, Zhongshan Hospital, Fudan University, Shanghai, China

\section{Keywords}

Chronic thromboembolic pulmonary hypertension $\cdot$ Heart rate recovery at one minute $\cdot$ Severity $\cdot$ Prognosis

\begin{abstract}
Background: Attenuated heart rate recovery at 1 min (HRR1) was demonstrated to correlate with poor prognosis in patients with pulmonary arterial hypertension, whereas its role in patients with chronic thromboembolic pulmonary hypertension (CTEPH) remains unclear. Objectives: The aim of this study was to investigate the correlations between HRR1 and functional status, echocardiography, hemodynamics, and prognosis of CTEPH. Methods: We retrospectively enrolled patients with CTEPH who underwent right heart catheterization and cardiopulmonary exercise test between June 2014 to October 2020 in Fuwai hospital. The primary outcome was clinical worsening. Linear regression was performed to assess the association between HRR1 and established markers of CTEPH severity. Receiver operating characteristic curve analysis was performed to determine the optimal cutoff of HRR1. Cox regression models were used to assess the association between HRR1 and clinical worsening. Results: A to-
\end{abstract}

karger@karger.com www.karger.com/res

Karger $\stackrel{\text { ' }}{5}$

GOPEN ACCESS
(C) 2021 The Author(s)

Published by S. Karger AG, Basel

This is an Open Access article licensed under the Creative Commons Attribution-NonCommercial-4.0 International License (CC BY-NC) (http://www.karger.com/Services/OpenAccessLicense), applicable to the online version of the article only. Usage and distribution for commercial purposes requires written permission. tal of 211 patients with CTEPH were included in the study. After adjusting for confounders, HRR1 positively correlated with 6-min walk distance, mixed venous oxygen saturation, and peak oxygen consumption, and negatively correlated with NT-proBNP, pulmonary vascular resistance, and ventilatory equivalent of carbon dioxide. Compared with patients with HRR $1 \geq 16$ beats, patients with HRR $1<16$ beats had approximately a 3 -fold risk of experiencing clinical worsening and the risk escalated with time. Conclusion: HRR1 could reflect disease severity and was independently associated with prognosis in patients with CTEPH. @ 2021 The Author(s).

Published by S. Karger AG, Basel

\section{Introduction}

Chronic thromboembolic pulmonary hypertension (CTEPH) is featured by progressive increase of pulmonary arterial pressure (PAP), which would eventually lead to right heart failure and death [1]. Survival of patients with CTEPH is unfavorable [2]. In the 2015 Euro-

Qi Jin, Xin Li and Yi Zhang contributed equally to this work.
Correspondence to:

Qin Luo, luoqin2009@163.com

Zhihong Liu, zhihongliufuwai@163.com 
pean Society of Cardiology/European Respiratory Society guidelines for the diagnosis and treatment of pulmonary hypertension $(\mathrm{PH})$, a set of noninvasive parameters reflecting exercise capacity and cardiac function, including 6-min walk distance (6MWD), oxygen consumption at peak exercise, and World Health Organization Functional Class (WHO FC) have been used to predict the 1 -year survival of patients with pulmonary arterial hypertension (PAH) [1]. However, none of these could reflect autonomic nerve impairment, which has been demonstrated to play an important role in the development of $\mathrm{PH}$ [3].

Autonomic nerve function impairment refers to sympathetic nerve overactivation and blunted parasympathetic nerve function. Compared with complex and expensive examinations like baroreflex sensitivity quantification, heart rate recovery is a simple and reliable tool to assess autonomic nerve function, which is defined as decrease of heart rate within the following minutes after exercise cession $[4,5]$. Patients with compromised autonomic nerve function had attenuated heart rate recovery. There is a large number of evidence suggesting that attenuated heart rate recovery is a powerful prognostic biomarker in various populations, including coronary artery disease [6], heart failure [7], and asymptomatic individuals with increased risk of coronary artery disease [8]. Recently, several studies have also demonstrated that heart rate recovery at $1 \mathrm{~min}$ (HRR1) was associated with exercise capacity, right heart function, and prognosis in patients with PAH (group I PH) $[9,10]$. Unfortunately, the role of HRR1 in CTEPH (group IV PH) remains unclear. Moreover, most of the previous studies concerning the relationship between PAH and HRR1 were limited to small sample size $[10,11]$ and lack of representativeness. In the present study, we aimed to investigate the correlations between HRR1 and functional status, echocardiography, hemodynamics, and prognosis of CTEPH in a retrospective cohort.

\section{Method}

\section{Study Design and Population}

This retrospective cohort study was conducted in Fuwai hospital, Chinese Academy of Medical Sciences (Beijing, China). Consecutive patients diagnosed with CTEPH in accordance with current guidelines $[1,12]$ between June 2014 and October 2020 were eligible. Inclusion criteria were: (1) patients underwent right heart catheterization (RHC); (2) patients underwent cardiopulmonary exercise testing (CPET) and the time interval between CPET and RHC was $<3$ months. Patients were excluded if they received $\beta$ blockers or any other medicines that interfere heart rate and au- tonomous nerve function. The following clinical data were collected via an electronic medical record system by 2 independent reviewers: demographics, echocardiographic, hemodynamic, and CPET-derived parameters.

\section{Right Heart Catheterization}

RHC was performed as previously described [13]. A 7F SwanGanz catheter was inserted into right femoral vein after local anesthesia and subsequently reached pulmonary artery. Hemodynamic parameters including mixed venous oxygen saturation, right atrial pressure and right ventricular pressure, PAP, pulmonary artery wedge pressure and heart rate were measured. Pulmonary vascular resistance was calculated according to standard formulas [14].

\section{Cardiopulmonary Exercise Testing}

CPET was performed as previously described [13]. After 3-min rest and subsequent 3-min warm-up, work rate increased gradually (5-30 W/min) according to estimated exercise tolerance until volitional exhaustion or symptom limitation. CPET-derived parameters including pulmonary function, oxygen consumption, heart rate, blood pressure, oxygen pulse, and end-tidal partial pressure of carbon dioxide were measured. HRR1 was calculated as the difference of heart rate between the end of CPET and 1 min after cessation of exercise. Peak oxygen consumption ( $\left.\mathrm{VO}_{2} @ P e a k\right)$ was defined as the highest 30 's average of oxygen consumption in the last minute of exercise $[15,16]$.

\section{Follow-Up and Outcome}

Patients were followed up regularly through telephone call or outpatient examination until occurrence of outcome or end of the study, namely, December 2020. The outcome of the current study was clinical worsening, which was defined as occurrence of any of the following adverse events: death, rehospitalization due to heart failure or progression of $\mathrm{PH}$, lung transplant, and escalation of $\mathrm{PAH}$ therapy. Time to clinical worsening was the time from date of CPET to the occurrence of outcome or the end of follow-up. End-point events were adjudicated by 2 senior clinicians. Any discordance was resolved by the supervisors (Q.L. and Z.L.).

\section{Statistical Analysis}

Continuous data were presented as mean \pm standard deviation and categorical data were presented as number or percentage. For continuous variables, according to the distribution of data, independent sample $t$ test or nonparametric test was used to identify the difference between patients with and without clinical worsening. For categorical parameters, $\chi^{2}$ test or Fisher exact test was used where appropriate. Associations between HRR1 and other parameters were evaluated by multivariate linear regression to adjust for confounders. Receiver operator characteristic curve analysis was performed to determine the optimal cutoff of HRR1, which was 16 beats. Kaplan-Meier method was used to determine differences in clinical worsening events between patients with HRR1 above or below 16 beats, and curves were compared with the log-rank test. Univariate Cox analysis was used to identify potential risk factors of clinical worsening. Subsequently, data of statistical or clinical significance were included into multivariate Cox model. Linearity assumption of variables was examined by restricted cubic splines. Proportional hazards assumption of variables in multivariate Cox regression was evaluated by Schoenfeld residuals and variables that violated proportional hazards assumption entered as time-depen- 
dent variable in the multivariate Cox regression model. A 2-sided $p$ value $<0.05$ was considered statistically significant. Statistical analysis was performed with SPSS 25.0 (IBM SPSS Corp., Armonk, NY, USA), R (version 4.0.5; R Foundation for Statistical Computing, Vienna, Austria), and GraphPad Prism 8 (GraphPad Software, LaJolla, CA, USA).

\section{Results}

\section{Patient Enrollment}

From June 2014 to October 2020, a total of 211 patients with CTEPH who underwent RHC and CPET were included into the study (Fig. 1), among whom $43.6 \%$ of patients were female, and the median follow-up period was $13.9 \pm 8.7$ months. During the follow-up, 6 patients died and 39 patients rehospitalized for heart failure or progression of PH or escalation of PAH therapy.

\section{Differences in Variables between Patients with and without Clinical Worsening}

Demographic parameters did not differ between the 2 groups, whereas patients experiencing clinical worsening had significantly worse WHO FC (III/IV 51.1\% vs. $27.1 \%$, $p=0.002)$ and were less likely to receive aggressive treatment (PEA: $6.7 \%$ vs. 22.3\%, BPA:31.1\% vs. $47.0 \%$, Medicine: $51.1 \%$ vs. $26 \%, p<0.003$, Table 1 ).

Hemodynamic, echocardiographic, and CPET-derived parameters at baseline were similar between the 2 groups (Tables 2, 3). Distinctively, patients with clinical worsening had significantly lower HRR1 (20.0 \pm 9.4 beats vs. $14.6 \pm 9.6$ beats, $p<0.001)$ compared with survivors without clinical worsening. Besides lower HRR1, patients with clinical worsening had higher heart rate at rest (76.9 $\pm 12.3 \mathrm{beat} / \mathrm{min}$ vs. $81.8 \pm 14.1 \mathrm{beat} / \mathrm{min}, p=0.021)$ and lower peak heart rate $(128.0 \pm 19.8 \mathrm{beat} / \mathrm{min}$ vs. $122.2 \pm$ $21.5 \mathrm{beat} / \mathrm{min}, p=0.089$ ) compared with patients without clinical worsening (Table 3 ).

\section{Correlation between HRR1 and Established Disease Severity Markers of CTEPH}

After adjusting for confounders, HRR1 had significant correlation with functional status, echocardiography, and hemodynamics (Table 4). Specifically, HRR1 was positively correlated with $6 \mathrm{MWD}(\beta=0.23, p=0.007)$, mixed venous oxygen saturation $(\beta=0.30, p<0.001)$, and $\mathrm{VO}_{2} @$ Peak $(\beta=0.46, p<0.001)$; it was negatively associated with $\mathrm{N}$ terminal-pro brain natriuretic peptide (NT-proB$\mathrm{NP})(\beta=-0.33, p<0.001)$, pulmonary vascular resistance $(\beta=-0.33, p<0.001)$, and ventilatory equivalent of carbon dioxide $(\beta=-0.44, p<0.001)$.

The Value of HRR1 in CTEPH

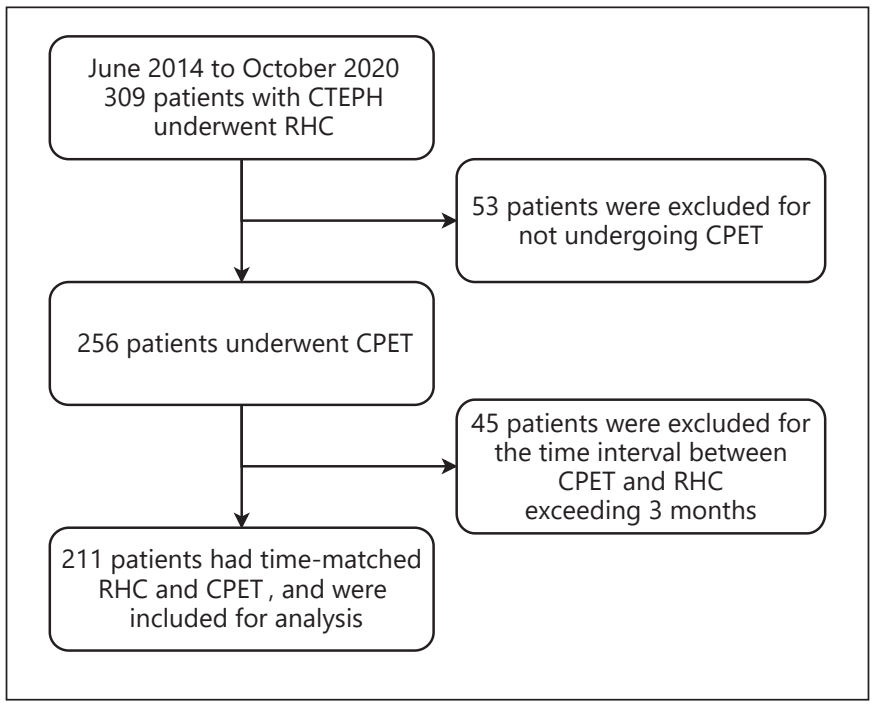

Fig. 1. Flow diagram of participant enrollment and exclusion. CPET, cardiopulmonary exercise testing; CTEPH, chronic thromboembolic pulmonary hypertension; RHC, right heart catheterization.

\section{Survival Analysis}

Receiver operating characteristic was performed in order to identify the best cutoff of HRR1, and HRR1 was dichotomized into HRR $<16$ and $\geq 16$ beats (online suppl. Fig. 1; see www.karger.com/doi/10.1159/000520314 for all online suppl. material). In the current cohort, $37.4 \%$ of patients with CTEPH had HRR $1<16$ beats. Compared to those with $\mathrm{HRR} 1 \geq 16$ beats, patients with $\mathrm{HRR} 1<16$ beats had significantly worse cardiac function, hemodynamics, and exercise capacity, as reflected by worse WHO FC, higher NT-proBNP, higher mean PAP, elevated pulmonary vascular resistance, more compromised $\mathrm{VO}_{2} @ P e a k$, lower oxygen pulse, worse ventilation efficiency, and shorter 6MWD (online suppl. Tables 1, 2).

According to Kaplan-Meier curve, patients with HRR1 $<16$ beats had significantly worse survival and shorter time to clinical worsening than patients with HRR $1 \geq 16$ beats ( $p=0.027$, Fig. 2$)$. Univariate Cox analysis identified that HRR $1 \geq 16$ beats, WHO FC, treatment, workload at peak exercise, respiratory exchange ratio at peak exercise, minute ventilation and heart rate at peak exercise were associated with clinical worsening (Table 5). HRR1 correlated linearly with outcome and the risk of clinical worsening decreased as HRR1 increased according to restricted cubic spline (online suppl. Fig. 2). As shown in Figure 2, survival curves of HRR $1<16$ and $\geq 16$ beats intersected before the first 10 months, indicating that HRR1 
Table 1. Demographic features of patients with and without CW

\begin{tabular}{|c|c|c|c|}
\hline Variables & Non-CW $(n=166)$ & $\mathrm{CW}(n=45)$ & $p$ value \\
\hline Age, years & $54.0 \pm 12.0$ & $55.2 \pm 15.2$ & 0.563 \\
\hline Female, $n(\%)$ & $73(44.0)$ & $19(42.2)$ & 0.833 \\
\hline Drink, $n(\%)$ & $33(20.0)$ & $6(13.6)$ & 0.336 \\
\hline Smoke, $n(\%)$ & $48(29.1)$ & $10(22.7)$ & 0.402 \\
\hline Hyperlipidemia, n (\%) & $31(18.8)$ & $10(22.7)$ & 0.559 \\
\hline Diabetes mellitus, $n$ (\%) & $11(6.7)$ & $6(13.6)$ & 0.133 \\
\hline Hypertension, $n$ (\%) & $36(21.7)$ & $13(28.9)$ & 0.310 \\
\hline \multicolumn{4}{|l|}{ WHO FC } \\
\hline I-II, n (\%) & $96(57.8)$ & $16(35.6)$ & \multirow{2}{*}{0.002} \\
\hline III-IV, n (\%) & $45(27.1)$ & $23(51.1)$ & \\
\hline NT-proBNP, ng/L & $882.2(198.6,1,838.8)$ & $1,316.0(262.7,2,239.8)$ & 0.508 \\
\hline \multicolumn{4}{|l|}{ Treatment } \\
\hline PEA, $n(\%)$ & $37.0(22.3)$ & $3.0(6.7)$ & \multirow{4}{*}{0.003} \\
\hline $\mathrm{BPA}, n(\%)$ & $78.0(47.0)$ & $14.0(31.1)$ & \\
\hline Medicine, $n(\%)$ & $43.0(26.0)$ & $23.0(51.1)$ & \\
\hline None, $n(\%)$ & $8(4.7)$ & $5(11.1)$ & \\
\hline
\end{tabular}

Data are presented as mean \pm standard deviation, median (range) or number (percentage). BPA, balloon pulmonary angioplasty; CW, clinical worsening; Medicine, pulmonary hypertension specific therapy; NT-proBNP, N-terminal pro-brain natriuretic peptide; PEA, pulmonary endarterectomy; WHO FC, World Health Organization functional class; 6MWD, 6-min walk distance; WHO FC, was not available in all patients.
Table 2. Hemodynamic and echocardiographic parameters of patients with and without CW

\begin{tabular}{llll}
\hline Variables & Non-CW $(n=166)$ & CW $(n=45)$ & $p$ value \\
\hline $\begin{array}{lll}\text { Echocardiography } \\
\text { LA, mm }\end{array}$ & $33.2 \pm 5.4$ & $35.2 \pm 8.3$ & 0.057 \\
LVED, mm & $40.2 \pm 6.6$ & $40.1 \pm 7.1$ & 0.941 \\
EF, \% & $63.7 \pm 5.5$ & $63.6 \pm 5.4$ & 0.881 \\
RVED, mm & $33.3 \pm 7.2$ & $33.8 \pm 7.3$ & 0.701 \\
sPAP, mm Hg & $87.8 \pm 24.7$ & $84.9 \pm 26.0$ & 0.499 \\
TRV, m/s & $4.37 \pm 0.70$ & $4.40 \pm 0.71$ & 0.799 \\
TRPG, mm Hg & $78.8 \pm 23.3$ & $75.55 \pm 23.6$ & 0.423 \\
TAPSE, mm & $16.4 \pm 3.8$ & $14.7 \pm 4.9$ & 0.073 \\
Hemodynamics & & & \\
S、O $\%$ \% & $68.9 \pm 6.3$ & $67.5 \pm 6.6$ & 0.185 \\
mRAP, mm Hg & $6.0(3.0,9.0)$ & $6.0(3.5,11.5)$ & 0.095 \\
mPAP, mm Hg & $48.8 \pm 11.7$ & $48.6 \pm 12.9$ & 0.926 \\
PAWP, mm Hg & $8.4 \pm 3.9$ & $9.77 \pm 3.6$ & 0.053 \\
Cl, L/min/m ${ }^{2}$ & $3.0 \pm 0.8$ & $3.0 \pm 0.8$ & 0.840 \\
PVR, wood units & $9.1 \pm 4.0$ & $9.2 \pm 4.3$ & 0.838 \\
\hline
\end{tabular}

Data are presented as mean \pm standard deviation or median (range). CW, clinical worsening; $\mathrm{Cl}$, cardiac index; $\mathrm{EF}$, ejection fraction; $\mathrm{LA}$, left atrium dimension; LVED, left ventricular end-diastolic diameter; mPAP, mean pulmonary artery pressure; mRAP, mean right atrial pressure; PAWP, pulmonary artery wedge pressure; PVR, pulmonary vascular resistance; RVED, right ventricular end-diastolic diameter; SPAP, systolic pulmonary artery pressure; $\mathrm{S}_{\mathrm{v}} \mathrm{O}_{2}$, mixed venous oxygen saturation; TAPSE, tricuspid annular plane systolic excursion; TRPG, tricuspid regurgitation pressure gap; TRV, tricuspid regurgitation velocity. 
Table 3. Exercise measurements of patients with and without CW
Fig. 2. Kaplan-Meier curves for patients with $\operatorname{HRR} 1<16$ and $\geq 16$ beats. HRR 1 , heart rate recovery at $1 \mathrm{~min}$.

\begin{tabular}{|c|c|c|c|}
\hline Variables & Non-CW $(n=166)$ & $\mathrm{CW}(n=45)$ & $p$ value \\
\hline 6MWD, m & $388.7 \pm 114.3$ & $345.9 \pm 131.4$ & 0.071 \\
\hline \multicolumn{4}{|l|}{ CPET } \\
\hline HRR1, beats & $20.0 \pm 9.4$ & $14.6 \pm 9.6$ & $<0.001$ \\
\hline Workload@Peak, W & $76.1 \pm 30.9$ & $61.9 \pm 25.6$ & 0.005 \\
\hline RER@Peak & $1.05 \pm 0.12$ & $1.01 \pm 0.09$ & 0.051 \\
\hline BR@Peak,*\% & $47.8 \pm 16.0$ & $49.7 \pm 12.3$ & 0.840 \\
\hline $\mathrm{VO}_{2} @$ Peak, mL/min/kg & $12.9 \pm 3.6$ & $11.9 \pm 2.6$ & 0.077 \\
\hline VE@Peak, L/min & $46.0 \pm 14.1$ & $40.1 \pm 12.2$ & 0.011 \\
\hline HR@Rest, beat/min & $76.9 \pm 12.3$ & $81.8 \pm 14.1$ & 0.021 \\
\hline HR@Peak, beat/min & $128.0 \pm 19.8$ & $122.18 \pm 21.5$ & 0.089 \\
\hline SBP@Peak, mm Hg & $137.0 \pm 28.7$ & $133.0 \pm 31.7$ & 0.443 \\
\hline DBP@Peak, mm Hg & $85.3 \pm 20.3$ & $86.0 \pm 25.0$ & 0.853 \\
\hline $\mathrm{VO}_{2} / \mathrm{HR} @$ Peak, mL/beat & $6.9 \pm 2.3$ & $6.36 \pm 1.9$ & 0.147 \\
\hline $\mathrm{P}_{\mathrm{ET}} \mathrm{CO}_{2} @$ Peak, mm Hg & $23.7 \pm 6.0$ & $23.6 \pm 6.0$ & 0.889 \\
\hline $\mathrm{P}_{\mathrm{ET}} \mathrm{O}_{2} @$ Peak, mm Hg & $124.3 \pm 6.8$ & $123.8 \pm 6.2$ & 0.661 \\
\hline VE/VCO ${ }_{2} @ A T$ & $47.2 \pm 10.1$ & $49.0 \pm 11.5$ & 0.299 \\
\hline VE $/ \mathrm{VCO}_{2}$ slope & $47.5 \pm 15.6$ & $47.6 \pm 18.5$ & 0.968 \\
\hline $\mathrm{VO}_{2} / \mathrm{WR}$ slope, $\mathrm{mL} / \mathrm{min} / \mathrm{W}$ & $4.1 \pm 3.7$ & $4.3 \pm 2.6$ & 0.785 \\
\hline Lowest VE/ $\mathrm{VCO}_{2}$ & $43.6 \pm 9.2$ & $44.1 \pm 7.5$ & 0.768 \\
\hline
\end{tabular}

Data are presented as mean \pm standard deviation or median (range). Bold values are statistically significant. BR, breathing reserve; $C W$, clinical worsening; $C P E T$, cardiopulmonary exercise testing; DBP@Peak, diastolic blood pressure at peak exercise; HR, heart rate; HRR1, heart rate recovery at $1 \mathrm{~min} ; \mathrm{MVV}$, maximum ventilatory volume; $\mathrm{P}_{\mathrm{ET}} \mathrm{CO}_{2} @$ Peak, end-tidal partial pressure of carbon dioxide at peak exercise; $\mathrm{P}_{\mathrm{ET}} \mathrm{O}_{2} @ P e a k$, end-tidal partial pressure of oxygen at peak exercise; RER@Peak, respiratory exchange ratio at peak exercise; SBP@ Peak, systolic blood pressure at peak exercise; 6MWD, 6-min walk distance; VE@Peak, minute ventilation at peak exercise; VE $/ \mathrm{VCO}_{2} @ \mathrm{AT}$, minute ventilation/carbon dioxide production at anaerobic threshold; $\mathrm{VO}_{2} @ P e a k$, oxygen consumption at peak exercise; $\mathrm{VO}_{2} /$ HR@Peak, oxygen consumption/heart rate at peak exercise; $\mathrm{VO}_{2} / \mathrm{WR}$, oxygen consumption/ work rate. $* \mathrm{BR}=(\mathrm{MVV}-\mathrm{VE} @ P$ eak $) / \mathrm{MVV} \times 100 \%$.

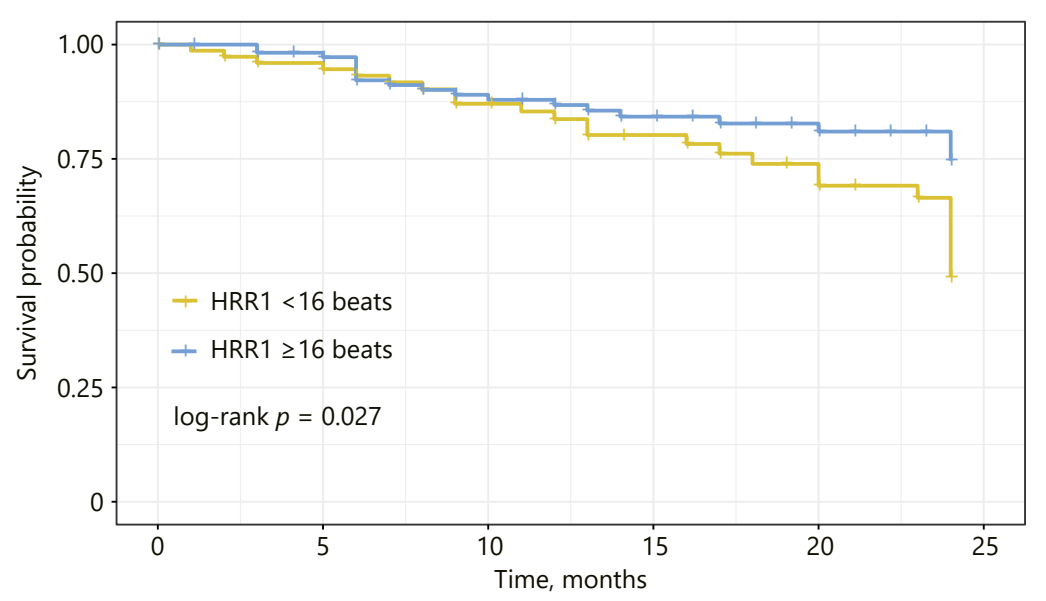

Number at risk

HRR $1<16$ beats 79

HRR $1 \geq 16$ beats 132

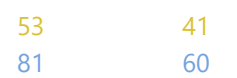

60

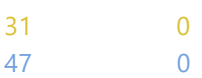


Table 4. Association between HRR1 and established markers of CTEPH severity

\begin{tabular}{llr}
\hline Variables* & $\beta$ & $p$ value \\
\hline WHO FC & -0.17 & $\mathbf{0 . 0 2 3}$ \\
6MWD & 0.23 & $\mathbf{0 . 0 0 7}$ \\
NT-proBNP & -0.41 & $\mathbf{0 . 0 0 1}$ \\
TAPSE & 0.26 & $\mathbf{0 . 0 0 6}$ \\
$\mathrm{S}_{\mathrm{V}}$ & 0.30 & $\mathbf{0 . 0 0 1}$ \\
mPAP & -0.22 & $\mathbf{0 . 0 0 2}$ \\
$\mathrm{Cl}$ & 0.25 & $<\mathbf{0 . 0 0 1}$ \\
$\mathrm{PVR}$ & -0.33 & $\mathbf{< 0 . 0 0 1}$ \\
VO $_{2} @$ Peak & 0.46 & $<\mathbf{0 . 0 0 1}$ \\
VO $_{2} /$ HR@Peak & 0.33 & $<\mathbf{0 . 0 0 1}$ \\
VO $_{2} /$ WR slope & 0.33 & $<\mathbf{0 . 0 1 0}$ \\
VE/VCO $_{2} @ A T$ & -0.44 & $<\mathbf{0 . 0 0 1}$ \\
\hline
\end{tabular}

Bold values are statistically significant. $\mathrm{Cl}$, cardiac index; $\mathrm{CTEPH}$, chronic thromboembolic pulmonary hypertension; HRR1, heart rate recovery at $1 \mathrm{~min}$; $\mathrm{mPAP}$, mean pulmonary artery pressure; NTproBNP, N-terminal pro-brain natriuretic peptide; PVR, pulmonary vascular resistance; $\mathrm{S}_{\mathrm{v}} \mathrm{O}_{2}$, mixed venous oxygen saturation; TAPSE, tricuspid annular plane systolic excursion; VE/ $/ \mathrm{CO}_{2} @ A T$, minute ventilation/carbon dioxide production at anaerobic threshold; $\mathrm{VO}_{2} @$ Peak, oxygen consumption at peak exercise; $\mathrm{VO}_{2} / \mathrm{HR} @$ Peak, peak oxygen consumption/heart rate at peak exercise; $\mathrm{VO}_{2} / \mathrm{WR}$, oxygen consumption/workrate;WHOFC, World Health Organization functional class; 6MWD, 6-min walk distance. ${ }^{*}$ All the variables were adjusted for age, sex, height, weight, diabetes mellitus by multivariate linear regression.

violated proportional hazards assumption, which was further verified by Schoenfeld residual (online suppl. Table 3). Accordingly, follow-up time was stratified into 2 intervals, namely within 10 months and over 10 months. HRR $1 \geq 16$ beats entered multivariate Cox model as a time-dependent covariate. Multivariate Cox analysis further confirmed that HRR $1 \geq 16$ beats was independently associated with prognosis after adjusting for confounders. In the first 10 months, the risk of clinical worsening was comparable between patients with HRR $1<16$ and $\geq 16$ beats. When follow-up time exceeded 10 months, patients with HRR1 $<16$ beats had approximately a 3 -fold risk of experiencing clinical worsening compared with patients with HRR1 $\geq 16$ beats (Table 6 ).

\section{Discussion}

In the fields of $\mathrm{PH}$, most of the previous studies concerning autonomic nerve function were limited to $\mathrm{PAH}$ (group I PH) [3, 17, 18]. Our findings extend this knowledge to CTEPH (group IV PH). The most important find-
Table 5. Univariate Cox regression of risks for clinical worsening in patients with CTEPH

\begin{tabular}{|c|c|c|c|}
\hline Variables & $\mathrm{HR}$ & $95 \% \mathrm{Cl}$ & $p$ value \\
\hline Female & 0.78 & $0.43-1.41$ & 0.416 \\
\hline 6MWD & 1.00 & $0.99-1.01$ & 0.106 \\
\hline WHO FC & 2.30 & $1.22-4.36$ & 0.010 \\
\hline Treatment & & & 0.002 \\
\hline None/Medicine & 1.52 & $0.58-4.01$ & 0.393 \\
\hline PEA/Medicine & 0.18 & $0.05-0.61$ & 0.005 \\
\hline BPA/Medicine & 0.41 & $0.21-0.80$ & 0.008 \\
\hline $\mathrm{S}_{\mathrm{v}} \mathrm{O}_{2}$ & 0.98 & $0.93-1.03$ & 0.395 \\
\hline mRAP & 1.05 & $0.99-1.11$ & 0.132 \\
\hline mPAP & 1.00 & $0.98-1.03$ & 0.769 \\
\hline $\mathrm{Cl}$ & 0.91 & $0.61-1.34$ & 0.617 \\
\hline PVR & 1.03 & $0.95-1.12$ & 0.434 \\
\hline Workload@Peak & 0.99 & $0.97-0.99$ & 0.015 \\
\hline RER@Peak & 0.05 & $0.003-0.832$ & 0.037 \\
\hline HRR $1 \geq 16$ beats & 0.53 & $0.29-0.95$ & 0.033 \\
\hline VO $_{2} @$ Peak & 0.93 & $0.85-1.03$ & 0.182 \\
\hline VE@Peak & 0.98 & $0.95-0.99$ & 0.036 \\
\hline HR@Peak & 0.98 & $0.97-0.99$ & 0.045 \\
\hline $\mathrm{VO}_{2} / \mathrm{HR} @$ Peak & 0.94 & $0.81-1.09$ & 0.396 \\
\hline $\mathrm{P}_{\mathrm{ET}} \mathrm{CO}_{2} @$ Peak & 1.01 & $0.96-1.05$ & 0.835 \\
\hline VE/VCO $2 @ A T$ & 1.02 & $0.99-1.05$ & 0.219 \\
\hline $\mathrm{VO}_{2} /$ WR slope & 1.02 & $0.90-1.16$ & 0.765 \\
\hline
\end{tabular}

Bold values are statistically significant. BPA, balloon pulmonary angioplasty; $\mathrm{Cl}$, cardiac index; $\mathrm{CTEPH}$, chronic thromboembolic pulmonary hypertension; HR, heart rate; HRR1, heart rate recovery at 1 min; Medicine, pulmonary hypertension specific therapy; mPAP, mean pulmonary artery pressure; mRAP, mean right atrial pressure; $\mathrm{PEA}$, pulmonary endarterectomy; $\mathrm{P}_{\mathrm{ET}} \mathrm{CO}_{2} @$ Peak, end-tidal partial pressure of carbon dioxide at peak exercise; $P V R$, pulmonary vascular resistance; RER, respiratory exchange ratio; $\mathrm{S}_{\mathrm{v}} \mathrm{O}_{2}$, mixed venous oxygen saturation; VE@Peak, minute ventilation at peak exercise; VE/ $\mathrm{VCO}_{2} @ \mathrm{AT}$, minute ventilation/carbon dioxide production at anaerobic threshold; $\mathrm{VO}_{2} @$ Peak, oxygen consumption at peak exercise; $\mathrm{VO}_{2} / \mathrm{HR} @$ Peak, oxygen consumption/heart rate at peak exercise; $\mathrm{VO}_{2} / \mathrm{WR}$, oxygen consumption/work rate; 6MWD, 6-min walk distance; WHO FC, World Health Organization functional class.

ing of our study is that easily available and noninvasive HRR1 is a strong predictor of clinical worsening in patients with CTEPH. We also found that autonomic nerve impairment was highly prevalent in CTEPH patients with over one-third of patients with CTEPH having a HRR1 $<16$ beats.

\section{Cutoff Value of HRR1}

Peçanha et al. [4] summarized the cutoff value of heart rate recovery in predicting mortality in various populations. For instance, HRR $1<12$ beats and heart rate recovery at $2 \mathrm{~min}<24$ beats were associated with the increased rate 
Table 6. Time-dependent Cox regression of risks for clinical worsening in patients with CTEPH

\begin{tabular}{|c|c|c|c|}
\hline Variables & $\mathrm{HR}$ & $95 \% \mathrm{Cl}$ & $p$ value \\
\hline \multicolumn{4}{|l|}{ Model 1} \\
\hline Treatment & & & $<0.001$ \\
\hline None/Medicine & 1.90 & $0.71-5.10$ & 0.202 \\
\hline PEA/Medicine & 0.18 & $0.05-0.60$ & 0.005 \\
\hline BPA/Medicine & 0.37 & $0.19-0.73$ & 0.004 \\
\hline HRR $1 \geq 16$ beats (time $<10$ months) & 0.82 & $0.34-1.97$ & 0.650 \\
\hline HRR $1 \geq 16$ beats (time $\geq 10$ months) & 0.29 & $0.13-0.67$ & 0.004 \\
\hline \multicolumn{4}{|l|}{ Model 2} \\
\hline Treatment & & & 0.002 \\
\hline None/Medicine & 2.03 & $0.52-7.99$ & 0.310 \\
\hline PEA/Medicine & 0.22 & $0.06-0.74$ & 0.014 \\
\hline BPA/Medicine & 0.37 & $0.19-0.73$ & 0.004 \\
\hline HRR $1 \geq 16$ beats (time $<10$ months) & 1.08 & $0.41-2.85$ & 0.873 \\
\hline HRR $1 \geq 16$ beats (time $\geq 10$ months) & 0.41 & $0.17-0.98$ & 0.044 \\
\hline Workload@Peak & 0.99 & $0.97-1.00$ & 0.081 \\
\hline \multicolumn{4}{|l|}{ Model 3} \\
\hline Treatment & & & 0.003 \\
\hline None/Medicine & 1.68 & $0.53-5.26$ & 0.376 \\
\hline PEA/Medicine & 0.24 & $0.07-0.83$ & 0.024 \\
\hline BPA/Medicine & 0.36 & $0.17-0.74$ & 0.006 \\
\hline HRR $1 \geq 16$ beats (time $<10$ months) & 1.04 & $0.41-2.62$ & 0.932 \\
\hline HRR $1 \geq 16$ beats (time $\geq 10$ months) & 0.29 & $0.11-0.77$ & 0.013 \\
\hline WHOFC & 1.64 & $0.83-3.25$ & 0.153 \\
\hline \multicolumn{4}{|l|}{ Model 4} \\
\hline Treatment & & & 0.001 \\
\hline None/Medicine & 2.19 & $0.65-7.36$ & 0.205 \\
\hline PEA/Medicine & 0.26 & $0.07-0.89$ & 0.032 \\
\hline BPA/Medicine & 0.36 & $0.17-0.74$ & 0.005 \\
\hline HRR $1 \geq 16$ beats (time $<10$ months) & 1.23 & $0.48-3.19$ & 0.666 \\
\hline HRR $1 \geq 16$ beats (time $\geq 10$ months) & 0.36 & $0.13-0.98$ & 0.046 \\
\hline WHOFC & 1.66 & $0.83-3.29$ & 0.150 \\
\hline HR@Peak & 0.99 & $0.97-1.01$ & 0.156 \\
\hline \multicolumn{4}{|l|}{ Model 5} \\
\hline Treatment & & & 0.001 \\
\hline None/Medicine & 1.87 & $0.59-5.96$ & 0.291 \\
\hline PEA/Medicine & 0.24 & $0.07-0.84$ & 0.025 \\
\hline BPA/Medicine & 0.33 & $0.16-0.69$ & 0.003 \\
\hline HRR $1 \geq 16$ beats (time $<10$ months) & 1.21 & $0.47-3.14$ & 0.695 \\
\hline HRR $1 \geq 16$ beats (time $\geq 10$ months) & 0.34 & $0.12-0.93$ & 0.035 \\
\hline WHOFC & 1.65 & $0.82-3.29$ & 0.159 \\
\hline RER@Peak & 0.13 & $0.01-3.34$ & 0.219 \\
\hline \multicolumn{4}{|l|}{ Model 6} \\
\hline Treatment & & & 0.003 \\
\hline None/Medicine & 1.77 & $0.55-5.66$ & 0.337 \\
\hline PEA/Medicine & 0.25 & $0.07-0.87$ & 0.029 \\
\hline BPA/Medicine & 0.37 & $0.18-0.77$ & 0.008 \\
\hline HRR $1 \geq 16$ beats (time $<10$ months) & 1.11 & $0.43-2.89$ & 0.827 \\
\hline HRR $1 \geq 16$ beats (time $\geq 10$ months) & 0.31 & $0.11-0.85$ & 0.023 \\
\hline WHOFC & 1.59 & $0.80-3.18$ & 0.190 \\
\hline VE@Peak & 0.99 & $0.97-1.02$ & 0.575 \\
\hline
\end{tabular}

Bold values are statistically significant. BPA, balloon pulmonary angioplasty; CTEPH, chronic thromboembolic pulmonary hypertension; HR, heart rate; HRR1, heart rate recovery at $1 \mathrm{~min}$; Medicine, pulmonary hypertension specific medicine; PEA, pulmonary endarterectomy; RER@Peak, respiratory exchange ratio at peak exercise; VE, minute ventilation; WHO FC, World Health Organization functional class. 
of mortality in patients with heart failure $[7,19]$. The cutoff value in the current study was 16 beats, which was in accordance with the study conducted by Minai et al. [9] and Yang et al. [20]. The discrepancy in cutoff value may be attributable to diversity in study population, sample size, and different methods of measuring heart rate recovery.

\section{HRR1 Was Associated with Established Markers of CTEPH Severity}

In line with previous studies [10,21], we also found significant correlation between HRR1 and parameters having been applied for risk stratification and prognosis prediction in patients with $\mathrm{PAH}$, including NT-proBNP, mixed venous oxygen saturation,6MWD, and $\mathrm{VO}_{2} @ P e a k$, indicating that HRR1 could also reflect disease progression.

\section{HRR1 Was Associated with Prognosis of CTEPH}

Although hemodynamics and exercise capacity at baseline were comparable between patients with and without clinical worsening, patients who experienced clinical worsening had significantly lower baseline HRR1 than survivors without events, indicating that autonomic nerve dysfunction was more evident than hemodynamics and exercise capacity impairment. In other words, HRR1 could be a more sensitive prognostic predictor than parameters reflecting pulmonary hemodynamics and exercise tolerance, including mean PAP, 6MWD, WHO FC, and NT-proBNP, which was in line with work by Minai et al. [21] They found that HRR $1<16$ beats measured after 6MWD test could predict clinical worsening more accurately than $6 \mathrm{MWD}$ or BNP $>100 \mathrm{pg} / \mathrm{mL}$.

Consistent with previous studies $[9,10,21]$, we found that HRR1 correlated independently with long-term prognosis after adjusting for confounders. Patients with CTEPH who had impaired HRR1 were more prone to experience clinical worsening than those with preserved HRR1 and the risk exaggerated with time. Previous studies have found that HRR1 could predict clinical worsening in patients with chronic obstructive pulmonary disease [22], congestive heart failure, idiopathic pulmonary fibrosis [23], idiopathic PAH [9], and connective tissue disease-associated $\mathrm{PH}$ [21]. The current study filled the knowledge gap in CTEPH.

Patients with $\mathrm{PAH}$ were reported to have overactivated renin-angiotensin-aldosterone system and sympathetic nerve function, which was reflected by increased angiotensin II and plasma norepinephrine concentration [24, 25]. Overactivated sympathetic nerve function and blunt parasympathetic nerve function were responsible for clinical deterioration and poor survival in patients with
PAH $[17,24]$. The underlying mechanism may be attributable to negative impact of overactivated sympathetic nerve function on pulmonary hemodynamics. Exaggerated sympathetic nerve activity elevates PAP and pulmonary vascular resistance, which outweighs relaxation effect of parasympathetic nerve. Secondary to increased right heart afterload, right ventricular enhances contraction to maintain cardiac output, leading to hypertrophied right ventricle. Secondary to right ventricular compression as well as leftward septal displacement, left heart function is also compromised and chronotropic incompetence occurs. This may explain why HHR 1 is associated with severity and prognosis of CTEPH.

\section{Limitation}

The current study had inherent limitation of retrospective study and 32\% CTEPH patients were not included in the study due to lack of CPET results. Therefore, we compared baseline features of included and excluded patients (online suppl. Tables 4,5) and found that excluded patients had worse hemodynamics and more severe cardiac impairment than included patients, which could be understandable, because patients with advanced $\mathrm{PH}$ were too weak to perform CPET. Therefore, the prevalence of heart rate recovery impairment as well as the hazard ratio of decreased HRR1 in CTEPH patients might be underestimated in the current study. Additionally, CPET and RHC were not performed simultaneously in all patients, which might lead to inconsistency between hemodynamics and CPET results. However, we limited the time interval to 3 months, where the state of patients was relatively stable. Moreover, the median follow-up was 14 months, therefore the time interval between RHC and CPET was negligible. The current study was conducted in a single center and the conclusion might not be generalized to the whole CTEPH population. However, Fuwai hospital is the national cardiovascular center of China and our patients were from all over the country. Moreover, CTEPH is a rare disease with the estimated prevalence ranging from 19 to 50 per million according to different studies [1], and the current study had the largest sample size in the context of investigating the role of HRR1 in PH.

\section{Conclusion}

Autonomic nerve dysfunction was highly prevalent in CTEPH patients, which could be easily identified by attenuated HRR1. HRR1 was associated with established 
markers of CTEPH severity. Moreover, CTEPH patients with attenuated HRR1 had poorer prognosis and the risk of clinical worsening increased with time.

\section{Statement of Ethics}

The study was conducted according to the guidelines of the Declaration of Helsinki, and approved by the Ethics Committee of Fuwai Hospital, approval number [2019-1191]. Due to the retrospective nature of this study, the need to obtain written informed consent was waived by the Ethics Committee of Fuwai Hospital.

\section{Conflict of Interest Statement}

The authors have no conflicts of interest to declare.

\section{Funding Sources}

This work was supported by Beijing Municipal Science and Technology Project (Grant No. Z181100001718200), Beijing Municipal Natural Science Foundation (Grant No. 7202168), CAMS Innovation Fund for Medical Sciences (CIFMS) (2020-I2M-C\&T-
B-055), and the Capital's Funds for Health Improvement and Research (CFH) (2020-2-4033). The funding source(s) had no involvement in study design or in the collection, analysis, or interpretation of data or in the writing of the report or in the decision to submit the article for publication.

\section{Author Contributions}

Y.Z. and X.L. contributed to conceptualization; Q.J. contributed to methodology; X.L. contributed to software, writing - original draft preparation - and project administration; Z.Z. and L.Y. contributed to validation; Y.Z. contributed to formal analysis; A.D. and L.Y. contributed to investigation; Q.Z. and A.D. contributed to resources; X.L. and Q.Z. contributed to data curation; Y.Z. and Q.L. contributed to writing - review and editing; Z.Z. contributed to visualization; Q.J. and Z.L. contributed to supervision; Z.L. and Q.L. contributed to funding acquisition. All authors have read and agreed to the published version of the manuscript.

\section{Data Availability Statement}

All data generated or analyzed during this study are included in this manuscript.

\section{References}

1 Galie N, Humbert M, Vachiery JL, Gibbs S, Lang I, Torbicki A, et al. 2015 ESC/ERS guidelines for the diagnosis and treatment of pulmonary hypertension: the joint task force for the diagnosis and treatment of pulmonary hypertension of the European Society of Cardiology (ESC) and the European Respiratory Society (ERS): endorsed by: Association for European Paediatric and Congenital Cardiology (AEPC), International Society for Heart and Lung Transplantation (ISHLT). Eur Heart J. 2016 Jan 1;37(1):67119.

2 Kim NH, Delcroix M, Jais X, Madani MM, Matsubara H, Mayer E, et al. Chronic thromboembolic pulmonary hypertension. Eur Respir J. 2019 Jan;53(1):1801915.

3 Vaillancourt M, Chia P, Sarji S, Nguyen J, Hoftman N, Ruffenach G, et al. Autonomic nervous system involvement in pulmonary arterial hypertension. Respir Res. 2017 Dec 4;18(1):201.

4 Peçanha T, Silva-Júnior ND, Forjaz CL. Heart rate recovery: autonomic determinants, methods of assessment and association with mortality and cardiovascular diseases. Clin Physiol Funct Imaging. 2014 Sep; 34(5):327-39.

5 Fecchio RY, Brito L, Leicht AS, Forjaz CLM, Peçanha T. Reproducibility of post-exercise heart rate recovery indices: a systematic review. Auton Neurosci. 2019 Nov;221:102582.
6 Lachman S, Terbraak MS, Limpens J, Jorstad $\mathrm{H}$, Lucas C, Scholte Op Reimer W, et al. The prognostic value of heart rate recovery in patients with coronary artery disease: a systematic review and meta-analysis. Am Heart J. 2018 May; 199:163-9.

7 Nanas S, Anastasiou-Nana M, Dimopoulos S, Sakellariou D, Alexopoulos G, Kapsimalakou S, et al. Early heart rate recovery after exercise predicts mortality in patients with chronic heart failure. Int J Cardiol. 2006 Jun 28;110(3):393-400.

8 Morshedi-Meibodi A, Larson MG, Levy D, O'Donnell CJ, Vasan RS. Heart rate recovery after treadmill exercise testing and risk of cardiovascular disease events (The Framingham Heart Study). Am J Cardiol. 2002 Oct 15;90(8):848-52.

9 Minai OA, Gudavalli R, Mummadi S, Liu X, McCarthy K, Dweik RA. Heart rate recovery predicts clinical worsening in patients with pulmonary arterial hypertension. Am J Respir Crit Care Med. 2012 Feb 15;185(4):400-8

10 Ramos RP, Arakaki JS, Barbosa P, Treptow E, Valois FM, Ferreira EV, et al. Heart rate recovery in pulmonary arterial hypertension: relationship with exercise capacity and prognosis. Am Heart J. 2012 Apr;163(4):580-8.
11 Inagaki T, Terada J, Yahaba M, Kawata N, Jujo T, Nagashima K, et al. Heart rate and oxygen saturation change patterns during 6-min walk test in subjects with chronic thromboembolic pulmonary hypertension. Respir Care. 2018 May;63(5):573-83.

12 Galiè N, Hoeper MM, Humbert M, Torbicki A, Vachiery JL, Barbera JA, et al. Guidelines for the diagnosis and treatment of pulmonary hypertension: the task force for the diagnosis and treatment of pulmonary hypertension of the European Society of Cardiology (ESC) and the European Respiratory Society (ERS), endorsed by the International Society of Heart and Lung Transplantation (ISHLT). Eur Heart J. 2009;30(20):2493-537.

13 Jin Q, Luo Q, Yang T, Zeng Q, Yu X, Yan L, et al. Improved hemodynamics and cardiopulmonary function in patients with inoperable chronic thromboembolic pulmonary hypertension after balloon pulmonary angioplasty. Respir Res. 2019 Nov 8;20(1):250.

14 Maron BA, Brittan EL, Hess E, Waldo SW, Barón AE, Huang S, et al. Pulmonary vascular resistance and clinical outcomes in patients with pulmonary hypertension: a retrospective cohort study. Lancet Respir Med. 2020 Sep;8(9):873-84

15 Tang Y, Luo Q, Liu Z, Ma X, Zhao Z, Huang $Z$, et al. Oxygen uptake efficiency slope predicts poor outcome in patients with idiopathic pulmonary arterial hypertension. J Am Heart Assoc. 2017 Jun 30;6(7). 
16 Cardoso FB, Antunes-Correa LM, Silva TQAC, Silva LM, Toledo C, Ribeiro VC, et al. Noninvasive imaging assessment of rehabilitation therapy in heart failure with preserved and reduced left ventricular ejection fraction (IMAGING-REHAB-HF): design and rationale. Ther Adv Chronic Dis. 2019; 10:2040622319868376.

17 Velez-Roa S, Ciarka A, Najem B, Vachiery JL, Naeije R, van de Borne P. Increased sympathetic nerve activity in pulmonary artery hypertension. Circulation. 2004 Sep 7; 110(10):1308-12.

18 Zhang H, Chen SL. Pulmonary artery denervation: update on clinical studies. Curr Cardiol Rep. 2019 Sep 5;21(10):124.

19 Lipinski MJ, Vetrovec GW, Gorelik D, Froelicher VF. The importance of heart rate recovery in patients with heart failure or left ventricular systolic dysfunction. J Card Fail. 2005 Oct;11(8):624-30.
20 Yang GL, Guo J, Pudasaini B, Yuan P, Gong $S G$, Wang L, et al. Value of heart rate recovery in female patients with pulmonary arterial hypertension due to systemic lupus erythematosus. Clin Respir J. 2019 Sep;13(9): 545-54.

21 Minai OA, Nguyen Q, Mummadi S, Walker E, McCarthy K, Dweik RA. Heart rate recovery is an important predictor of outcomes in patients with connective tissue disease-associated pulmonary hypertension. Pulm Circ. 2015 Sep;5(3):565-76.

22 Lacasse M, Maltais F, Poirier P, Lacasse Y, Marquis K, Jobin J, et al. Post-exercise heart rate recovery and mortality in chronic obstructive pulmonary disease. Respir Med. 2005 Jul;99(7):877-86.
23 Swigris JJ, Olson AL, Shlobin OA, Ahmad S, Brown KK, Nathan SD. Heart rate recovery after six-minute walk test predicts pulmonary hypertension in patients with idiopathic pulmonary fibrosis. Respirology. 2011 Apr;16(3):439-45.

24 Nootens M, Kaufmann E, Rector T, Toher C, Judd D, Francis GS, et al. Neurohormonal activation in patients with right ventricular failure from pulmonary hypertension: relation to hemodynamic variables and endothelin levels. J Am Coll Cardiol. 1995; 26(7):1581-5.

25 Hilzendeger AM, Shenoy V, Raizada MK, Katovich MJ. Neuroinflammation in pulmonary hypertension: concept, facts, and relevance. Curr Hypertens Rep. 2014;16(9): 469. 